\title{
Finite Groups Having Exactly 34 Elements of Maximal Order
}

\section{Zhangjia Han, Chao Yang}

School of Applied Mathematics, Chengdu University of Information Technology,Chengdu, China

\section{ABSTRACT}

Let $G$ be a finite group, $M(G)$ denotes the number of elements ofmaximal order of $G$. In this note a finite group $G$ with $M(G)$ $=34$ is determined.

\section{Indexing terms/Keywords}

Finite groups; Classification; Number of elements of maximal order; Thompson's Conjectur.

\section{Academic Discipline And Sub-Disciplines}

Mathematics

\section{SUBJECT CLASSIFICATION}

2010 Mathematics Subject Classification: 20D45, 20E34

\section{TYPE (METHOD/APPROACH)}

Mathematics research

\section{Council for Innovative Research}

Peer Review Research Publishing System

Journal: JOURNAL OF ADVANCES IN MATHEMATICS

Vol.11, No. 5

www.cirjam.com, editorjam@gmail.com 


\section{INTRODUCTION}

For a finite group $\mathrm{G}$, we denote by $\mathrm{M}(\mathrm{G})$ the number of elements of maximal order of $\mathrm{G}$, and the maximal element order in $\mathrm{G}$ by $\mathrm{k}=\mathrm{k}(\mathrm{G})$. There is atopic related to one of Thompson's Conjectures:

Thompson's Conjecture: Let $G$ be a finite group. For a positive integerd, define $G(d)=\mid\{x \in G \mid$ the order of $x$ is $d\} \mid$. If $S$ is a solvable group, $G(d)=S(d)$ for $d=1 ; 2 ; \ldots$, then $G$ is solvable.

Recently, some authors have investigated this topic in several articles(see[3], [7], [8], [9]). In particular, in [2] the authors gave a complete classfication of the finite group with $M(G)=30$, and the finite group with $M(G)=24$ are classified in [6]. In this paper, we consider a finite group $G$ satisfying $M(G)=34$. Our main result of this paper is:

Main Theorem: Suppose $\mathrm{G}$ is a finite group having exactly 34 elements of maximal order. Then $\mathrm{G}$ is solvable and $|\mathrm{G}|$ $=2^{\alpha} 3^{\beta}$, where $2 \leq \alpha \leq 7$, and $1 \leq \beta \leq 4$.

By the above theorem, we have:

Corollary: Thompson's Conjecture holds if $\mathrm{G}$ has exactly 34 elements of maximal order.

All groups considered are finite and all unexplained notations are standard and can be found in [4].

\section{Preliminaries}

The following lemma reveals the relationship of $M(G)$ and $k$.

Lemma 2.1 [9, Lemma 1] Suppose G has exactly $n$ cyclic subgroups of orderl, then the number of elements of order I (de -noted by $\left.n_{l}(G)\right)$ is $n_{l}(G)=n \phi(I)$, where $\phi(I)$ is the Euler function of $I$. In particular, if $n$ denotes the number

of cyclic subgroups of $G$ of maximal order $k$, then $M(G)=n \phi(k)$.

By above lemma, we have:

Lemma 2.2 If $M(G)=34$ and $k$ is maximal element order of $G$, thenpossible values of $n, k$ and $\phi(k)$ are given in following table:

\begin{tabular}{|c|c|c|}
\hline $\mathrm{n}$ & $\phi(\mathrm{k})$ & $\mathrm{k}$ \\
\hline 34 & 1 & 2 \\
\hline 17 & 2 & $3,4,6$ \\
\hline 2 & 17 & null \\
\hline 1 & 34 & null \\
\hline
\end{tabular}

In proving our main theorem, the following two results will be frequently used.

Lemma 2.3 [2, Lemma 6] If $k$ is prime, and the number of elements of maximal order $k$ is $m$, then $k$ divides $m+1$.

Lemma 2.4 [2, Lemma 8] If the number of elements of maximal order $\mathrm{k}$ is $\mathrm{m}$, then there exists a positive integer $\alpha$ such that $|\mathrm{G}|$ divides $\mathrm{mk}^{\alpha}$.

Lemma 2.5 [8, Lemma 2.5] Let $P$ be a $p$-group with order $p^{t}$, where $p$ is a prime, and $t$ is a positive integer. Suppose $b \in$ $Z(P)$, where $o(b)=p^{u}=k$ with $u$ a positive integer. Then $P$ has at least $(p-1) p^{t-1}$ elements of order $k$.

\section{Proof of Main Theorem}

By the hypothesis $M(G)=34$, then $k 6=2$ by Lemma 2.3, and and $k 6=4$ by [2, Corollary 2]. In the following we prove our theorem case by case for the remaining possible values of $k$.

Case $1 \mathrm{k}=3$. In this case $\mathrm{G}$ is a 3-group or a $\{2,3\}$-group. If $\mathrm{G}$ is a 3-group, then $\exp (\mathrm{G})=3$. By [5, Theorem 3.8.8], the number of 3-elements $M(G)$ of $G$ satisfies that $M(G) \equiv 4(\bmod 9)$, which contradicts with the fact $M(G)=34$. Hence $G$ is not a 3-group. If $\mathrm{G}$ is a $\{2,3\}$-group, then $\pi_{e}(\mathrm{G})=\{1,2,3\}$. By $[1$, Theorem] we know that $\mathrm{G}=\mathrm{N} \succ \mathrm{Q}$ is a Frobenius group, whe -re $\mathrm{N} \cong C_{3}^{t}, \mathrm{Q} \cong C_{2}$ or $\mathrm{N} \cong C_{2}^{2 t}, \mathrm{Q} \cong C_{3}$. Suppose that $\mathrm{Q} \cong C_{2}$. Then $\mathrm{N}$ is an elementary abelian 3-group, By [5, Theorem 3.8.8], we can get a contradiction. If $Q \cong C_{3}$, then the number of elements of order 3 is two, which contradicts to our assum ption. Thus $\mathrm{k} \neq 3$. 
Case $2 \mathrm{k}=6$. In this case $|\mathrm{G}|=2^{\alpha} 3^{\beta}$, where $\alpha>0$ and $\beta>0$ by Lemma 2.4. Let $\mathrm{x}$ be an element of order 6. Then $\left|\mathrm{C}_{G}(\mathrm{x})\right|$ $=2^{u} 3^{v}$. Since there exists no element of order 9 or 4 in $C_{G}(x)$, we have $v \leq 3$ and $u \leq 4$ by Lemma 2.5 . Since $G$ has exactly 17 cyclic subgroups of order 6 , we have $\left|G: N_{G}(<x>)\right|=1 ; 2 ; 3 ; 4 ; 6 ; 8 ; 9 ; 12$ or 16 . If there is an element $y$ of order 6 in $G$ such that $\left|G: N_{G}(<x>)\right|=9 ; 12$ or 16 , then there exists another element $z$ of order 6 in $G$ such that $\left|G: N_{G}(<x>)\right|=1 ; 2$; $3 ; 4 ; 6$ or 8 . That is to say, $G$ always has an element $x$ of order 6 such that $\mid G: N_{G}(\langle x>) \mid=1 ; 2 ; 3 ; 4 ; 6$ or 8 . Therefore $|G|$ $\mid 2^{7} 3^{4}$ since $|\mathrm{G}|=\left|\mathrm{G}: \mathrm{N}_{G}(<\mathrm{x}>)\right|\left|\mathrm{N}_{G}(<\mathrm{x}>): \mathrm{C}_{G}(\mathrm{x}) \| \mathrm{G}_{G}(\mathrm{x})\right|$. The Theorem is proved.

\section{ACKNOWLEDGMENTS}

This work is supported by the National Scienti' c Foundation of China(No: 11301426 and 11471055) and Scientific Research Foundation of SiChuan Provincial Education Department(No: 15ZA0181) and the Scientific Research Foundation of CUIT (No: J201418).

\section{REFERENCES}

[1] Brandl, R., Shi, W. J., Finite groups whose element orders are consecutive integers, J. Alg., 143(2)(1991), 388-400.

[2] Chen, G. Y., Shi,W. J., Finite groups with 30 elements of maximal order, Appl. Categor. Struct.16(2008), $239-247$.

[3] Du, X. L.,Jiang, Y. Y., On ${ }^{-}$nite groups with exact 4p elements of max-imal order are solvable, Chin. Ann. Math. 25A(5) (2004), 607-612 (inChinese).

[4] Gorenstein, D.,1968. Finite Groups, New York: Harper \& Row press.

[5] Huppert, B., 1967.Endliche Gruppern I, Springer-Verlag, Berlin/New York.

[6] Qinhui Jiang , Changguo Shao, Finite groups with 24 elements of maximal order, Front. Math. China, 5(4)(2010), 665678.

[7] Youyi Jiang, Finite groups with 2p2 elements of maximal order are solvable, Chin. Ann. Math., 21A(1)(2000), 6l-64(in Chinese).

[8] Youyi Jiang, A theorem of ' ${ }^{-}$nite groups with 18p elements having maximal order, Alg. Coll., 15(2)(2008), 317-329.

[9] Cheng Yang, Finite groups based on the numbers of elements of maximal order, Chin. Ann. Math.14A(5),(1993), 561567(in Chinese). 